\title{
First realization of a hybrid integrated diode laser in the visible spectral range
}

\author{
C.A.A. Franken ${ }^{1}$, A. van Rees ${ }^{1}$, Y. Fan', D. Geskus², R. Dekker², D.H. Geuzebroek ${ }^{2}$, C. Fallnich ${ }^{3}$, \\ P.J.M. van der Slot ${ }^{1}$ and K.-J. Boller ${ }^{1}$ \\ 1. Laser Physics and Nonlinear Optics Group, Faculty of Science and Technology, MESA+Institute of Nanotechnology, \\ University of Twente, Enschede, The Netherlands \\ 2. LioniX International B.V., Enschede, The Netherlands \\ 3. University of Münster, Institute of Applied Physics, Germany
}

Photonic integration of narrowband and tunable diode lasers in the visible range offers many advantages for applications in bio-photonics and metrology, e.g., for chip-based flow cytometry or portable atomic clocks. Extended cavity semiconductor lasers (ECDLs), based on bulk optical components, provide excellent spectral control, but suffer from an appreciable size, are susceptible to external perturbations, and lack the option for seamless integration into photonic circuits. Hybrid integrated diode lasers, using waveguide circuits for spectrally selective feedback, are small-sized and robust. These lasers provide wide tunability and sub-100-Hz intrinsic linewidths [1], but have only been realized at infrared wavelengths. Extending this concept towards the visible is not straightforward, as scattering and absorption losses typically increase at shorter wavelengths.

Here we demonstrate the first operation of a hybrid integrated diode laser operating in the visible. Optical amplification is provided by a diode amplifier, which is hybrid integrated with a $\mathrm{Si}_{3} \mathrm{~N}_{4}$ feedback circuit. Frequency selective feedback is provided by a pair of tunable microring resonators. To reduce the losses in this circuit, we exploit the weak mode confinement of a $25-\mathrm{nm}$ thin and $2-\mu \mathrm{m}$ wide $\mathrm{Si}_{3} \mathrm{~N}_{4}$ waveguide. We have operated the hybrid laser over a large spectral range of $11 \mathrm{~nm}$ around $685 \mathrm{~nm}$, where it delivers up to $5 \mathrm{~mW}$ output power. This first realization of a hybrid integrated diode laser in the visible range can provide a breakthrough in applications which rely on tunable and narrowband visible light sources integrated on a chip.

[1] Y. Fan et al., arXiv 1910.08141 (2019) 